\title{
INFLUÊNCIA DA ABSORÇÃO DE BIODIESEL NAS PROPRIEDADES DA BORRACHA NITRÍLICA
}

\author{
F. N. LINHARES ${ }^{1,2}$, M. KERSCH ${ }^{3}$, V. ALTSTAEDT ${ }^{3}$, C. R. G. FURTADO ${ }^{1}$ \\ ${ }^{1}$ Universidade do Estado do Rio de Janeiro, Instituto de Química \\ ${ }^{2}$ Bolsista da CAPES - Processo n ${ }^{\circ}$ 7709/13-7 \\ ${ }^{3}$ Universitaet Bayreuth, Lehrstuhl fuer Polymere Werkstoffe \\ E-mail para contato: fnlinhares@uerj.br; russi@uerj.br
}

\begin{abstract}
RESUMO - O biodiesel é um combustível com características semelhantes ao diesel comum, porém com menores níveis de emissão de poluentes. Entretanto, devido à sua composição química diferente, ele interage com diversos materiais de forma distinta do diesel. A borracha nitrílica (NBR) é bastante utilizada na indústria automotiva como matéria-prima de artefatos que estão em contato direto com diferentes combustíveis. Neste trabalho foi avaliada a resistência de borracha nitrílica com alto teor de acrilonitrila $(45 \%)$ a biodiesel puro oriundo de óleo de soja. Foram preparadas composições do elastômero com sistemas de vulcanização contendo diferentes teores de acelerador e de enxofre. Os testes de imersão foram realizados a $100^{\circ} \mathrm{C}$. Foi observada a variação de massa ao longo do tempo para definição do perfil de absorção do combustível no elastômero. Análises dinâmico mecânica (DMA), de calorimetria diferencial de varredura (DSC) e de dureza (Shore A) também foram realizadas.
\end{abstract}

\section{INTRODUÇÃO}

Biodiesel é quimicamente definido como uma mistura de mono-ésteres obtido, principalmente, a partir de óleos vegetais, podendo também ser produzido a partir de gorduras animais. Em 1937, foi patenteado na Bélgica, por G. Chavanne, o processo de transesterificação dos óleos vegetais (Suarez e Meneghetti, 2007; Mofijur et al., 2013). Em 1977, o processo industrial referente a obtenção de biodiesel foi patenteado pelo brasileiro Expedito Parente (Mojifur et al., 2013).

Apesar da semelhança das propriedades físicas entre o biodiesel e o diesel obtido de petróleo, ambos diferem significativamente quanto as suas composições químicas. O óleo diesel comum é obtido a partir da destilação de petróleo e de difícil caracterização. A destilação ocorre entre 170$350^{\circ} \mathrm{C}$ e as cadeias carbônicas variam de 9 a 12 carbonos (Agarwal et al., 2013), podendo também ser encontrados compostos alifáticos e aromáticos em sua composição (Lacava et al., 2005; Agarwal et $a l ., 2013)$. Biodiesel é formado por derivados ésteres de ácidos graxos e sua composição exata varia de acordo com a fonte utilizada, podem ser encontrados derivados de ácidos com cadeia carbônica alifática e insaturada, variando de 8 a 24 carbonos (Giakoumis, 2013).

A produção mundial de biodiesel cresceu substancialmente nos últimos anos, de 8 milhões de 


\section{9 a 22 de outubro de 2014 \\ Florianópolis/SC}

toneladas em 2007 para 20 milhões de toneladas em 2010 (Ong, et al., 2012). De acordo com o site Index Mundi (2013), os Estados Unidos da América lideram a produção mundial de biodiesel, seguido da Alemanha. Enquanto o Brasil aparece apenas na quarta colocação atrás da Argentina

Seu uso pode ser feito puro ou diluído sem a necessidade de alterações nos atuais motores Diesel. Em muitos países a comercialização de biodiesel misturado ao diesel comum é obrigatória. A concentração de biodiesel na mistura final do combustível é informada através de nomenclatura específica, definida como $B X$. Sendo, $X$ referente à porcentagem em volume de biodiesel misturado ao diesel comum: $B 2$ designa uma mistura biodiesel/diesel com $2 \%$ do biocombustível, sucessivamente, até $B 100$, referente ao seu uso puro.

Devido à alteração da composição química dos combustíveis faz-se necessário verificar a compatibilidade do biodiesel com os materiais já utilizados na indústria automotiva, além de entender como eles interagem. Na fabricação do motor são utilizados diversos materiais metálicos e nãometálicos, com os quais o combustível pode entrar constantemente em contato, como por exemplo: alumínio, ligas de cobre, aço inoxidável, elastômeros, plásticos, entre outros (Hasseb et al., 2011a).

O copolímero de butadieno e acrilonitrila, também conhecido como borracha nitrílica (NBR), é altamente polar e por isso é largamente utilizado na fabricação de artefatos que ficam em constante contato com combustíveis, óleos e solventes apolares.

Diferentes elastômeros também já foram testados em diversas condições frente a biodiesel de diferentes fontes vegetais (Trakarnpruk and Porntangjitlikit, 2008; Haseeb et al., 2010b; Haseeb et al., 2011 a, b; Chai et al., 2013; Linhares et al., 2013; Mofijur et al., 2013). Apesar de elastômeros fluorados terem se mostrados resistentes ao biodiesel, o seu alto custo pode trazer problemas quanto a sua aplicação em larga escala. Quanto à resistência da borracha nitrílica frente ao biodiesel, um aumento no teor de acrilonitrila em sua composição, induz a uma melhora na resistência do elastômero (Linhares et al., 2013).

\section{OBJETIVO}

Este trabalho tem como objetivo avaliar a resistência de amostras vulcanizadas do copolímero de butadieno e acrilonitrila (NBR), com alto teor de acrilonitrila, a biodiesel puro oriundo de óleo de soja, quanto à variação de massa, análises dinâmico mecânicas (DMA) e calorimetria diferencial de varredura (DSC).

\section{MATERIAIS E MÉTODOS}

\subsection{Processamento das composições elastoméricas}

Composições elastoméricas de borracha nitrílica com $45 \%$ de acrilonitrila, gentilmente cedida por Nitriflex S/A Indústria e Comércio, foram preparadas, com um sistema de cura a base de enxofre. As formulações foram elaboradas variando-se os teores de acelerador e de enxofre, conforme indicado na Tabela 1. As quantidades dos demais componentes foram mantidas iguais em todas as 
composições (em partes por 100 de borracha): borracha nitrílica (elastômero) - 100; óxido de zinco (ativador) - 3; ácido esteárico (ativador) - 1; negro de fumo (carga) - 40. As composições foram processadas em um misturador de cilindros aberto a $50^{\circ} \mathrm{C} \pm 5^{\circ} \mathrm{C}$. Foi utilizado negro de fumo SP 6630, gentilmente doado por Cabot Brasil Indústria e Comércio.

Tabela 1 - Teores de enxofre e de acelerador utilizados para o preparo das composições elastoméricas (quantidades em $\mathrm{phr}^{\mathrm{a}}$ )

\begin{tabular}{ccccc}
\hline $\begin{array}{c}\text { Componentes } \\
\text { da formulação }\end{array}$ & $\mathbf{1 S}$ & $\mathbf{2 S}$ & $\mathbf{5 S}$ & $\mathbf{6 S}$ \\
\hline $\begin{array}{c}\text { TMTD } \\
\text { (acelerador) }\end{array}$ & 1 & 1 & 3 & 3 \\
\hline $\begin{array}{c}\text { Enxofre } \\
\text { (agente de } \\
\text { vulcanização) }\end{array}$ & 0,5 & 1,5 & 0,5 & 1,5 \\
\hline $\begin{array}{c}\text { Relação entre } \\
\text { acelerador e } \\
\text { enxofre }\end{array}$ & 2,0 & 0,67 & 6,0 & 2,0 \\
\hline
\end{tabular}

\begin{tabular}{ccccc}
\hline $\begin{array}{l}\text { Tipo de } \\
\text { ligação } \\
\text { cruzada }\end{array}$ & $\begin{array}{c}\text { Semi-eficiente } \\
(\mathrm{SEV})\end{array}$ & $\begin{array}{c}\text { Convencional } \\
(\mathrm{CONV})\end{array}$ & Eficiente $(\mathrm{EV})$ & $\begin{array}{c}\text { Semi-eficiente } \\
\text { (SEV) }\end{array}$ \\
\hline $\begin{array}{l}\text { a - parts per hundred of rubber } \\
\text { tetrametiltiuram }\end{array}$ & partes para 100 partes de borracha); b - dissulfeto de
\end{tabular}

Pequenas amostras de cada composição foram analisadas em um reômetro de cavidade oscilante, MDPt, da TechPro, por $1 \mathrm{~h} \mathrm{a} 160^{\circ} \mathrm{C}$ para se obter o tempo ótimo de vulcanização. A vulcanização foi realizada em uma prensa hidráulica com os respectivos tempos de cura.

\subsection{Imersão}

Os testes de imersão foram realizados com biodiesel metílico puro de óleo de soja comprado da empresa alemã Analitik-Service Gesellschaft mbH. As imersões foram mantidas em estufa a $100^{\circ} \mathrm{C}$ por um período total de $190 \mathrm{~h}$. As principais propriedades físico-químicas do óleo combustível estão apresentadas na Tabela 2, juntamente com os limites regulamentares no Brasil e na Alemanha.

\subsection{Variação de massa}

Corpos de provas retangulares $(50 \mathrm{~mm} \times 10 \mathrm{~mm}$ e espessura máxima de 2,5mm) foram obtidos de cada composição, a fim de se obter o perfil da variação de massa das amostras ao longo do tempo. As massas das amostras foram medidas numa balança analítica com precisão de $0,1 \mathrm{mg}$, em diversos intervalos de tempo. As imersões foram realizadas em triplicata. 
Tabela 2 - Propriedades físico-químicas do biodiesel de soja utilizado

\begin{tabular}{ccccc}
\hline Propriedades & Valores & $\begin{array}{c}\text { Limites no } \\
\text { Brasil }^{\mathbf{a}}\end{array}$ & $\begin{array}{c}\text { Limites na } \\
\text { Alemanha }\end{array}$ & Unidade \\
\hline $\begin{array}{c}\text { Massa } \\
\text { específica }\end{array}$ & 884,9 & $850-900$ & $860-900$ & $\mathrm{~kg} / \mathrm{m}^{3}$ \\
\hline $\begin{array}{c}\text { Viscosidade } \\
\text { cinemática } \\
\left(40^{\circ} \mathrm{C}\right)\end{array}$ & 4,118 & $3,0-6,0$ & $3,5-5,0$ & $\mathrm{~mm}^{2} / \mathrm{s}$ \\
\hline Teor de água & 236 & 200 (máx.) $^{\text {Índice de }}$ & 500 (máx.) & $\mathrm{mg} / \mathrm{kg}$ \\
\hline acidez & 0,176 & 0,5 (máx.) & 0,5 (máx.) & $\begin{array}{c}\mathrm{mg} \mathrm{KOH} / \mathrm{g} \mathrm{de} \\
\text { óleo }\end{array}$ \\
\hline
\end{tabular}

a-Fonte: ANP, 2014

\subsection{Análise dinâmico mecânica (DMA)}

Para as análises dinâmico-mecânicas (DMA) das composições, foi utilizado um analisador RDA 3 , Rheometric Scientific. Corpos de provas retangulares com dimensões de $50 \mathrm{~mm}, 10 \mathrm{~mm}$ e espessura máxima de 2,5mm, das amostras não imersas e após $166 \mathrm{~h}$ de imersão, foram analisados em modo de deformação de tensão. As análises foram realizadas em frequência de $1 \mathrm{~Hz}$, na faixa de temperatura de $-50^{\circ} \mathrm{C}$ até $50^{\circ} \mathrm{C}$, com uma taxa de aquecimento de $3^{\circ} \mathrm{C} / \mathrm{min}$ e deformação de $0,3 \%$. A temperatura de transição vítrea $\left(T_{g}\right)$ foi calculada a partir dos pontos máximos das curvas do fator de amortecimento de cada composição.

\subsection{Calorimetria diferencial de varredura (DSC)}

Ensaios de calorimetria diferencial de varredura (DSC) foram realizados na faixa de temperatura de $-45^{\circ} \mathrm{C}$ até $40^{\circ} \mathrm{C}$ com taxa de aquecimento de $10^{\circ} \mathrm{C} / \mathrm{min}$. Os ensaios foram realizados em equipamento modelo DSC1 Star System, Mettler Toledo. As temperaturas de transição vítrea $\left(T_{g}\right)$ foram calculadas a partir da primeira derivada da curva de fluxo de calor (W/g) em função da temperatura.

\section{RESULTADOS E DISCUSSÃO}

\subsection{Variação de Massa}

Foram analisadas as variações de massa das amostras ao longo de $190 \mathrm{~h}(\approx 8$ dias $)$, incluindo os intervalos sugeridos pela norma ASTM D471 (22h, 46h, 166h), conforme mostra a Figura 1. Foi possível observar que a composição $5 S$ atinge o equilíbrio em torno de 50 horas, quando praticamente não mais se observou variação de massa ao longo do tempo. A quantidade de biocombustível absorvida permaneceu estagnada em torno 5,5\% em relação à massa inicial da amostra. Entretanto, para as demais amostras, foi observado que a massa continuou evoluindo sem que atingisse um ponto de saturação durante o tempo analisado.

De acordo com a Tabela 1, as ligações cruzadas presentes na composição $5 S$, que possui a maior 
relação entre quantidades de acelerador e de enxofre, são majoritariamente monossulfídicas, enquanto nas outras composições estão presentes di e polissulfídicas. Dessa forma, as cadeias poliméricas nessa composição estão mais próximas uma das outras, com menos volume vazio na estrutura tridimensional, o que resultou numa menor absorção de biodiesel.

Observou-se ainda que, para uma mesma quantidade de enxofre, um aumento no teor do acelerador utilizado (TMTD), induziu a uma menor absorção de biodiesel.

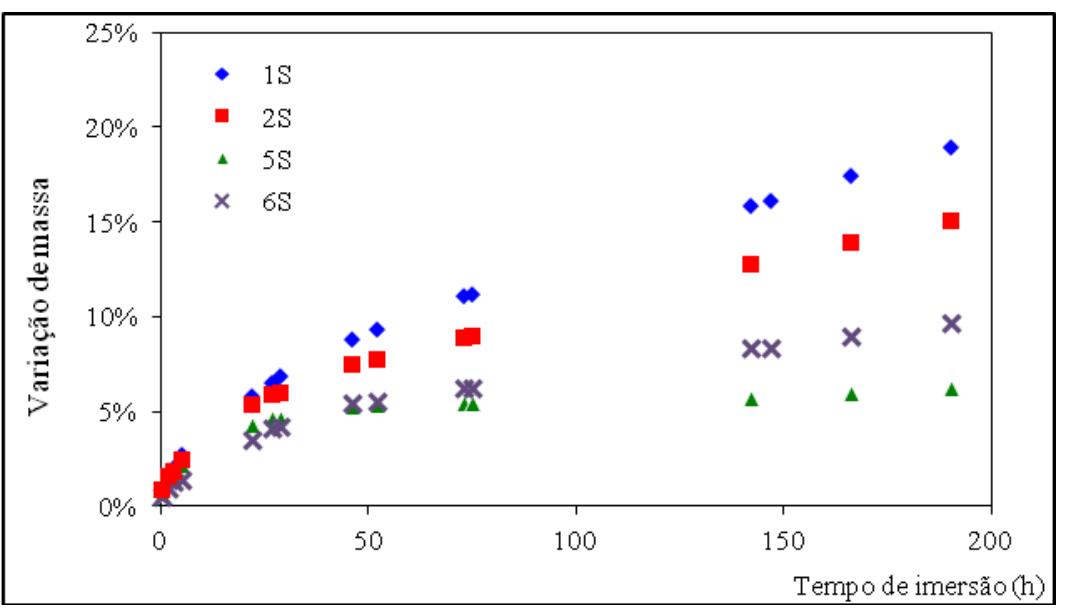

Figura 1 - Variação de massa das composições em função do tempo de imersão a $100^{\circ} \mathrm{C}$ em biodiesel de óleo de soja.

\subsection{Calorimetria diferencial de varredura (DSC)}

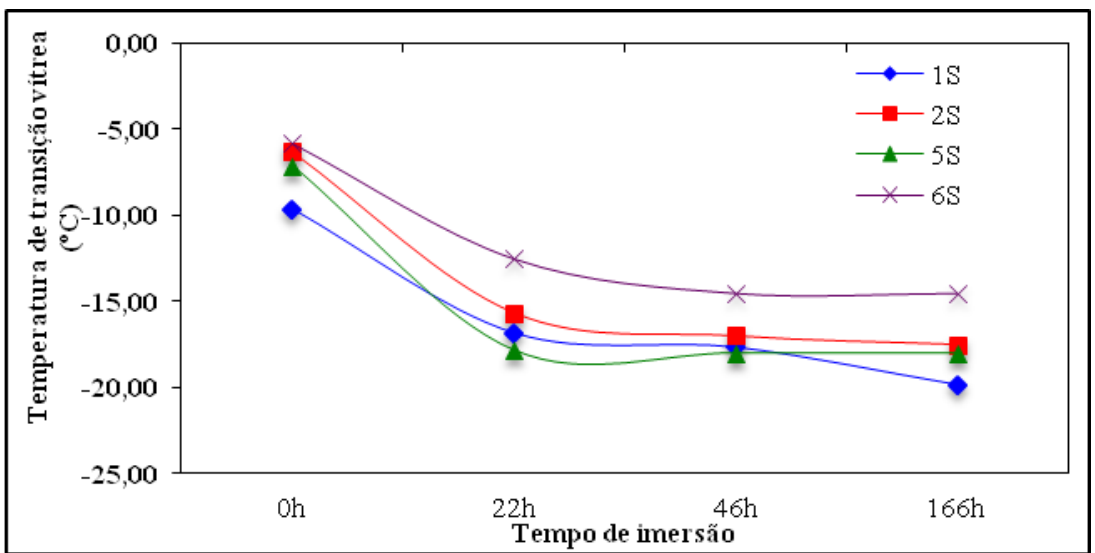

Figura 2 - Temperatura de transição vítrea $(T g)$ das composições em função do tempo de imersão a $100^{\circ} \mathrm{C}$ em biodiesel de óleo de soja.

As análises de DSC foram realizadas com amostras não imersas e após $22 \mathrm{~h}$, 46h e 166h de imersão a $100^{\circ} \mathrm{C}$ no biodiesel. Percebe-se que a absorção de biodiesel provocou uma diminuição da temperatura de transição vítrea $(T g)$ das amostras, conforme apresenta a Figura 2. Isso ocorre porque o biodiesel absorvido passa a atuar como plastificante dando mais mobilidade às moléculas 
poliméricas.

Após uma diminuição da $T g$ da amostra $5 S$ até $22 \mathrm{~h}$ de imersão, foi observado que a temperatura manteve-se constante ao longo do tempo (46h e 166h), pois, a partir de então, a amostra já se apresentava saturada de biodiesel. Esse fato corrobora o comportamento observado com a variação de massa da amostra ao longo do tempo. Todavia, a amostra $1 S$ apresentou um contínuo decréscimo da $T g$ ao longo do tempo, assim como a variação de massa, que durante o período analisado não apresentou um ponto de saturação.

\subsection{Análise dinâmico mecânicas (DMA)}
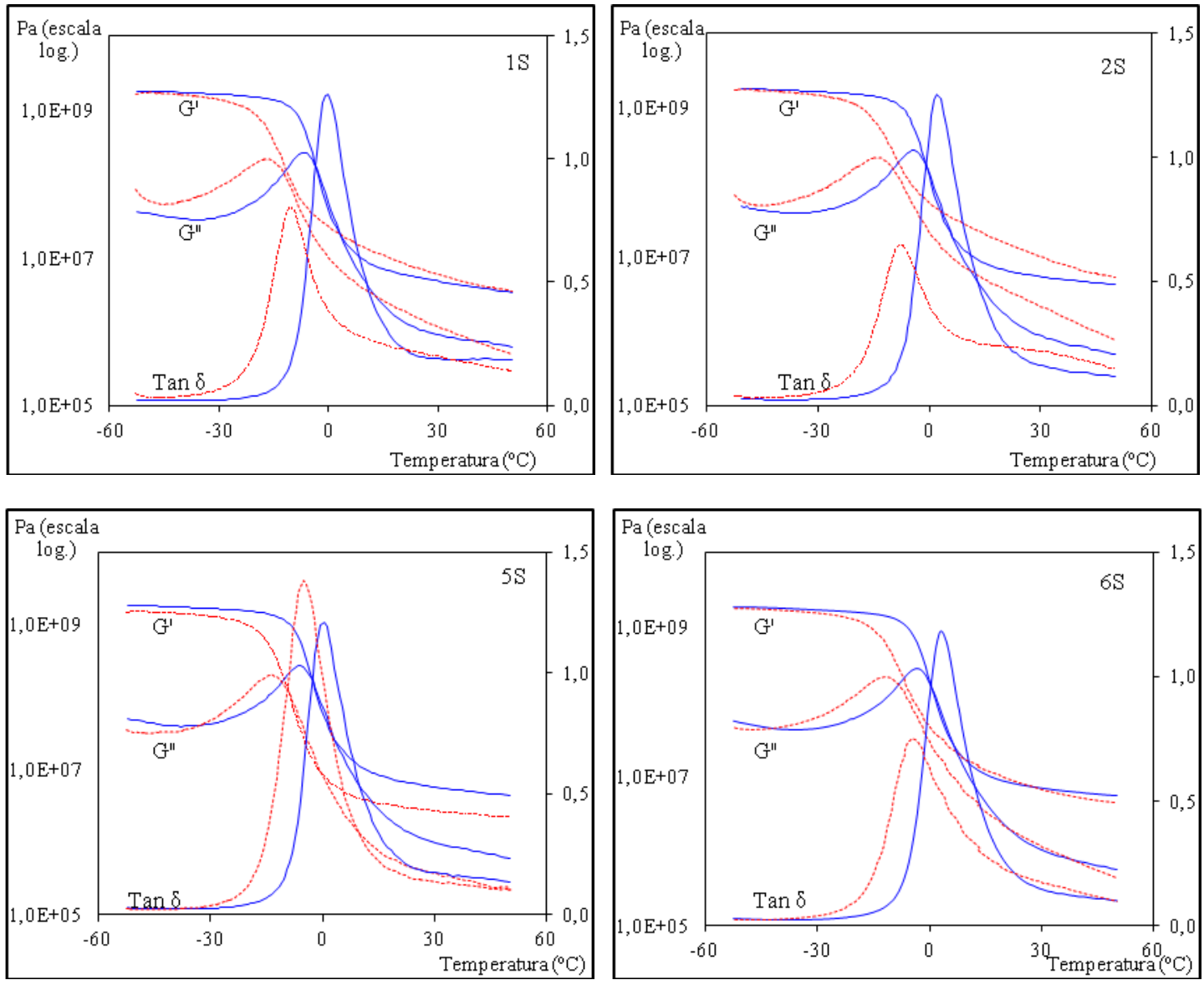

Figura 3 - Análise dinâmico mecânicas (DMA) das amostras não imersas (linha contínua azul) e após $166 \mathrm{~h}$ de imersão a $100^{\circ} \mathrm{C}$ em biodiesel de óleo de soja (linha tracejada vermelha). Módulo de armazenamento (G'), módulo de perda (G’) e fator de amortecimento $(\tan \delta)$.

As análises dinâmico mecânicas (DMA) estão expostas na Figura 3. Pôde-se perceber um discreto aumento do módulo de perda (G”) para temperaturas abaixo da transição vítrea. Nessas temperaturas, o óleo absorvido, encontrava-se ainda congelado, o que dificulta a movimentação das cadeias, aumentando a viscosidade das composições. Todavia, com o aumento da temperatura, o óleo 


\section{9 a 22 de outubro de 2014 \\ Florianópolis/SC}

torna-se fluido e passa a atuar como plastificante, facilitando a mobilidade das cadeias poliméricas e deslocando a $T_{g}$ para valores mais baixos do que o inicial.

Foi notado, que as amostras $1 S, 2 S$ e $6 S$ possuem um novo perfil após a imersão por 166h no biocombustível. O fator de amortecimento (tan $\delta$ ) apresentou, após a imersão, um pico e um posterior alongamento no formato de um ombro. Esse comportamento indica a forte atuação como plastificante do biodiesel quando absorvido pelo material. Essa absorção pode comprometer as propriedades mecânicas desse material por diminuir o entrelaçamento das cadeias poliméricas.

Entretanto, para a composição $5 S$, que absorveu a menor quantidade de biodiesel, o perfil dos parâmetros analisados praticamente não se alterou, apesar de levemente deslocado para temperaturas mais baixas.

\section{CONCLUSÕES}

Neste trabalho foi possível concluir que o biodiesel, ao ser absorvido pelas amostras de borracha nitrílica, atua como plastificante, permitindo maior mobilidade das cadeias poliméricas, alterando significativamente as propriedades originais do material de forma destrutiva. Entretanto, conclui-se também que modificando-se a formulação empregada nas composições, principalmente o sistema de cura, foi possível aumentar consideravelmente a resistência das amostras de borracha nitrílica, minimizando os efeitos nocivos do biocombustível para esse material. A formulação utilizada que promoveu a formação de ligações cruzadas predominantemente monossulfídica mostrou uma resistência superior as demais.

\section{AGRADECIMENTOS}

Os autores agradecem à Nitriflex S/A Indústria e Comércio pela doação das amostras elastoméricas; a Cabot Brasil Indústria e Comércio pela doação da amostra de negro de fumo; à Fundação Carlos Chagas Filho d Amparo à Pesquisa do Estado do Rio de Janeiro (FAPERJ) pelo auxílio financeiro do processo E-26/110.618/2011, à Dipl.-Chem.-Ing. Ute Kuhn pelo auxílio nas análises térmicas, ao Cléverson F. S. Gabriel pelo auxílio no preparo das amostras; ao Laboratório de Compostos de Borracha (IMA/UFRJ) pelas análises reométricas, e à Profa. Dra. Ana Maria F. de Sousa e à Profa. Dra. Marcia Christina A. M. Leite pelas valiosas discussões.

\section{REFERÊNCIAS}

ANP. Agência Nacional do Petróleo, Gás natural e Biocombustíveis. Disponível em: <http://www.anp.gov.br>. Acesso em: 30 mar 2014.

ARGAWAL, S.; CHHIBER V.K.; BHATNAGAR, A.K. Tribological behavior of diesel fuels and the effect of anti-wear additives. Fuel, v. 106, p. 21-29, 2013.

CHAI, A.B.; ANDRIYANA, A.; VERRON, E.; JOHAN, M.R. Mechanical characteristics of swollen elastomers under cyclic loading. Material \& Design v. 44, p. 566-572, 2013.

GIAKOUMIS, E.G. A statistical investigation of biodiesel physical and chemistry properties, and 
their correlation with the degree of unsaturation. Renewable Energy. v. 50, p. 858-878, 2013.

HASEEB, A.S.M.A; FAZAL, M.A.; JAHIRUL, M.I.; MASJUKI, H.H. Compatibility of automotive materials in biodiesel: A review. Fuel. v. 90, p. 922-931 $2011 \mathrm{a}$.

HASEEB, A.S.M.A.; MASJUKI, H.H.; SIANG, C.T.; FAZAL, M.A. Compatibility of elastomers in palm biodiesel. Renewable Energy. v.35, p.2356-2361, 2010b.

HASEEB, A.S.M.A.; JUN, T.S.; FAZAL, M.A.; MASJUKI, H.H. Degradation of physical properties of different elastomers upon exposure to palm biodiesel. Energy. v.36, p.1814-1819, 2011 b.

INDEX MUNDI, IndexMundi data portal. Disponível em: <http://www.indexmundi.com/energy.aspx?product=biodiesel $>$. Acesso em: 30 nov. 2013.

LACAVA, P. T.; CARVAlHO JUNIOR, J. A.; PIMENTA, A. P.; FERREIRA, M. A. Análise térmica do processo de incineração de resíduos aquosos com chamas enriquecidas. Tecnologia em Metalurgia e Materiais, v. 1, p. 16-21, 2005.

LINHARES, F.N.; CORRÊA, H.L.; KHALIL, C.N.; LEITE, M.C.A.M.; FURTADO, C.R.G. Study of the compatibility of nitrile rubber with Brazilian biodiesel. Energy, v.49, p.102-106, 2013.

MOFIJUR, M.; MASJUKI, H.H.; KALAM, M.A.; ATABANI, A.E.; SHAHABUDDIN, M. PALASH, S.M.; HAZRAT, M.A. Effect of biodiesel from various feedstocks on combustion characteristics, engine durability and materials compatibility: A review. Renewable and Sustainable Energy Reviews. v. 28, p. 441-455, 2013.

ONG, H.C.; MAHLIA, T.M.I.; MASJUKI, H.H.; HONNERY, D. Life cycle cost and sensitivity analysis of palm biodiesel production. Fuel, v. 98, p.131-139, 2012.

SUAREZ, P.A.Z.; MENEGHETTI, S.M.P. 70 aniversário do biodiesel em 2007: evolução histórica e situação atual no Brasil. Quím Nova, v. 30, p. 2068-2071 , 2007.

TRAKARNPRUK, W.; PORNTANGJITLIKIT, S. Palm oil biodiesel synthesized with potassium loaded calcined hydrotalcite and effect of biodiesel blend on elastomer properties. Renewable Energy. v. 33, p.1558-1563, 2008. 